\title{
NIPAL4/Ichthyin Is Expressed in the Granular Layer of Human Epidermis and Mutated in Two Pakistani Families with Autosomal Recessive Ichthyosis
}

\author{
Muhammad Wajid $^{a}$ Mazen Kurban $^{a}$ Yutaka Shimomura ${ }^{a}$ \\ Angela M. Christiano ${ }^{a, b}$ \\ Departments of a Dermatology and ${ }^{b}$ Genetics and Development, Columbia University, New York, N.Y., USA
}

\section{Key Words}

Ichthyosis · NIPAL4 • Atopic dermatitis • Filaggrin •

Autosomal recessive congenital ichthyosis

\begin{abstract}
Background: Autosomal recessive congenital ichthyosis (ARCI) can be divided into 3 types including lamellar ichthyosis (OMIM 242304), nonbullous congenital ichthyosiform erythroderma (OMIM 242100) and harlequin ichthyosis (OMIM 242500). The last type is uncommon since newborns with harlequin ichthyosis usually die shortly after birth. Several genes have been linked to $\mathrm{ARCl}$, but these represent only $60 \%$ of the known genetic causes of this condition. Methods: After having performed a linkage analysis, we analyzed the DNA of 2 consanguineous Pakistani families with ARCI for NIPAL4 mutations and performed in situ hybridization (ISH) for NIPAL4 mRNA in the epidermis. Results: The haplotype analysis revealed a linkage to chromosome 5 , and we identified a recurrent missense mutation, p.A176D, in affected individuals from both families. We also determined by ISH that NIPAL4 mRNA is highly expressed in the granular cell layer of the epidermis, consistent with the ARCI phenotype. Conclusion: Our results expand the spectrum of the clinical manifestations of the NIPAL4 gene and further extend our understanding of its molecular function.
\end{abstract}

Copyright $\odot 2009$ S. Karger AG, Basel
(C) 2009 S. Karger AG, Basel

Fax +4161306 1234 E-Mail karger@karger.ch www.karger.com www.karger.com/drm

\section{Introduction}

Autosomal recessive congenital ichthyosis (ARCI) can be clinically divided into 3 main categories. The first is lamellar ichthyosis (LI; OMIM 242304), the second nonbullous congenital ichthyosiform erythroderma (NBCIE; OMIM 242100) and the third harlequin ichthyosis (OMIM 242500), which is usually fatal shortly after birth. Because of this, several authors exclude harlequin ichthyosis from the classification and divide ARCI simply into 2 main subtypes.

In most cases of ARCI, infants are born with a collodion membrane. Clinically, LI is characterized by scales that are large, adherent, dark and pigmented, with an absence of skin erythema [1]. On the other hand, in NBCIE, the scales are fine and white on an erythematous background, although they are larger and grayish on the limbs $[2,3]$. Despite this clinical distinction, many cases of ARCI do not fit this classification, and patients frequently have manifestations of both conditions in addition to other features.

At the molecular level, mutations have been detected in $60 \%$ of the cases [4]. To date, 6 different genes have been implicated in the pathogenesis of ARCI, and these include: transglutaminase 1 (TGM1), ABCA12, 3-lipoxygenase (ALOXE3) and 12-lipoxygenase (ALOX12B), NIPA-like domain containing 4 (NIPAL4) and CYP4F22 [5].
Angela M. Christiano, $\mathrm{PhD}$

Department of Dermatology, Columbia University

College of Physicians and Surgeons

630 West 168th Street VC-1526, New York, NY 10032 (USA)

Tel. +1 212305 9565, Fax +1 212305 7391, E-Mail amc65@ columbia.edu 


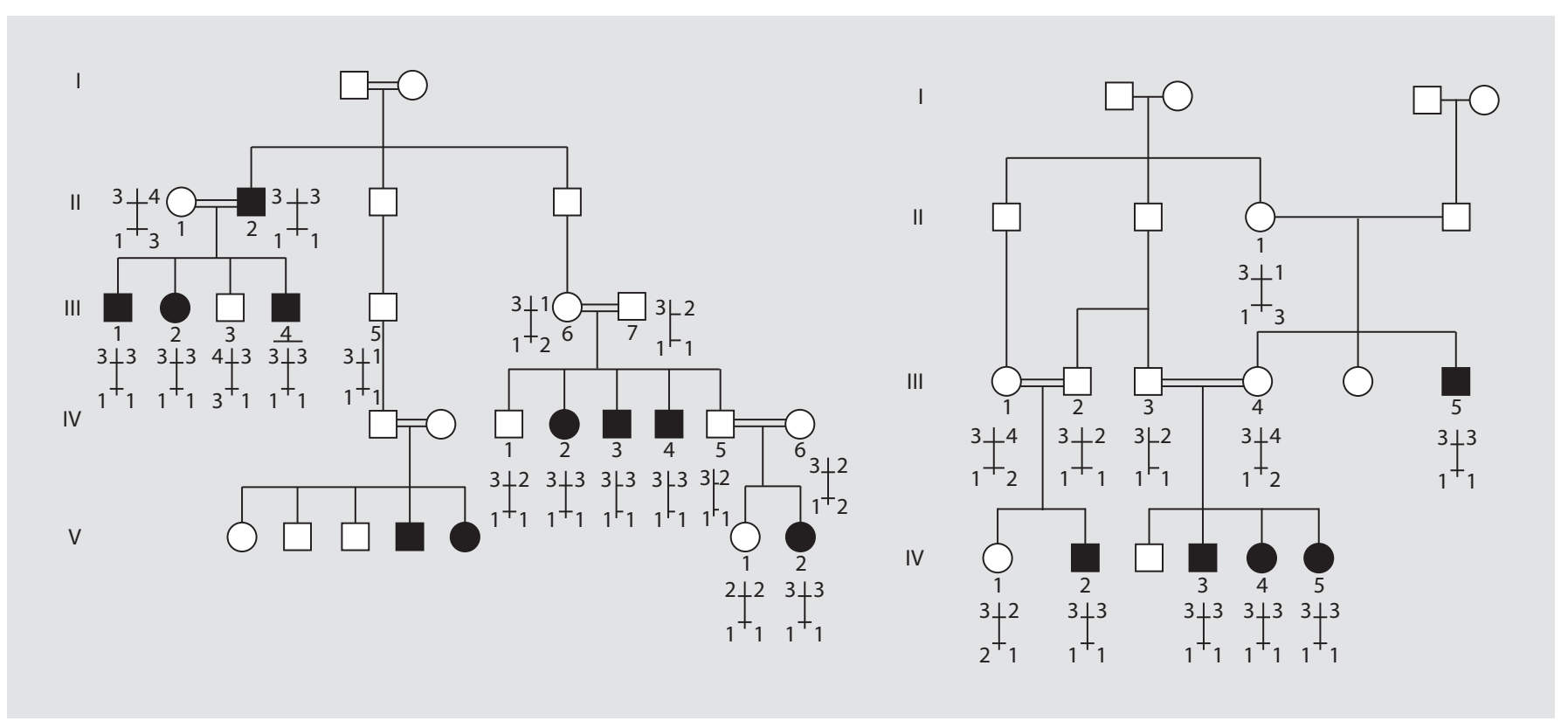

Fig. 1. Autosomal recessive inheritance is clearly seen in both pedigrees.

The pathophysiology of ARCI revolves around deficiencies in intercellular lipid [6]. The lipid that forms the 'cement' between corneocytes, and forms the epidermal barrier, originates from lamellar granules which are located in the granular cell layer $[7,8]$. The lamellar granules contain polar lipids including cholesterol sulfate, phospholipids, sphingomyelin and glucosylceramides that are the precursors of the intercellular lipids of the stratum corneum. Generally, lamellar granules encapsulate lipids from the cytosol of the keratinocytes by specialized transporters such as ABCA12 and deliver them to the cell membrane via direct fusion [7]. In addition, lamellar granules also contain other enzymes and proteases that are required for the normal desquamation of the epidermis $[7,9]$. Therefore, mutations in these proteins can cause a disturbance in barrier formation, leading to ARCI.

NIPAL4, also known as ichthyin, is composed of several transmembrane domains. Ichthyin-like proteins are localized on the plasma membrane, and share a homology to both transporters and G-protein-coupled receptors $[2,6]$. Ichthyin is thought to be a membrane receptor for the trioxilins $\mathrm{A} 3$ and $\mathrm{B} 3$, which are components of the hepoxilin pathway, but its function is yet to be determined.

To date, only a limited number of mutations in the NIPAL4 gene have been reported to be associated with
ARCI. These include 6 missense mutations, 2 splice site mutations and 1 nonsense mutation. Here, we analyzed 2 Pakistani families with clinical features of ARCI for mutations in the NIPAL4 gene. We also performed in situ hybridization (ISH) for NIPAL4 in the epidermis and localized it to the granular layer, consistent with the ARCI phenotype.

\section{Patients and Methods}

We analyzed 2 Pakistani families affected by an ichthyosiform disorder. After having obtained informed consent, we collected peripheral blood samples from members of these families in EDTA-containing tubes (under institutional approval and in adherence to the Declaration of Helsinki principles). Genomic DNA was isolated according to standard techniques. Both males and females were equally affected, suggestive of an autosomal recessive pattern of inheritance (fig. 1). On physical examination, dry skin was a common finding among all patients. Most young members in one family reported severe itching (fig. 2a), generalized multiple erythematous and nonerythematous crusted papules and hyperkeratotic plaques (fig. 2b), with fine whitish scales over the face (fig. 2c). Adult members of the same family had ectropion and severe whitish hyperkeratotic plaques over the face (fig. 2d), generalized semiadherent whitish-to-yellowish scales on an erythematous base (fig. 2e), joint contractures of the fingers and clubbing of the nails (fig. 2f). The second family also showed variation in the clinical presentation of its members with skin dryness and palmoplantar keratoderma (fig. $2 \mathrm{~g}$ ). Some patients showed 

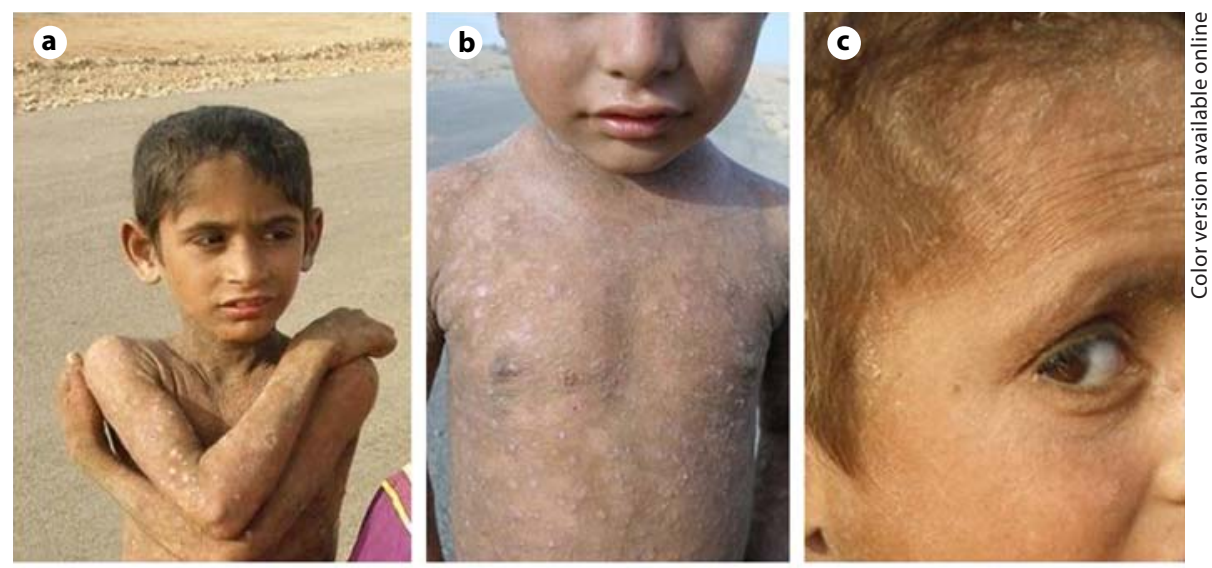

Fig. 2. a Severe itching in an affected individual. b Patient presenting with atopic dermatitis. c Fine whitish scales over the face of an affected individual. d Facial hyperkeratosis with ectropion, resembling LI. e Semiadherent yellowish-to-whitish scales overlying an erythematous base. f Joint contractures of the fingers with nail clubbing. g Palmar hyperkeratosis. h Fine whitish scales overlying an erythematous base, involving the neck and chest. Typical findings in NBCIE. i Brown reticulated ichthyosis involving the chest and neck.
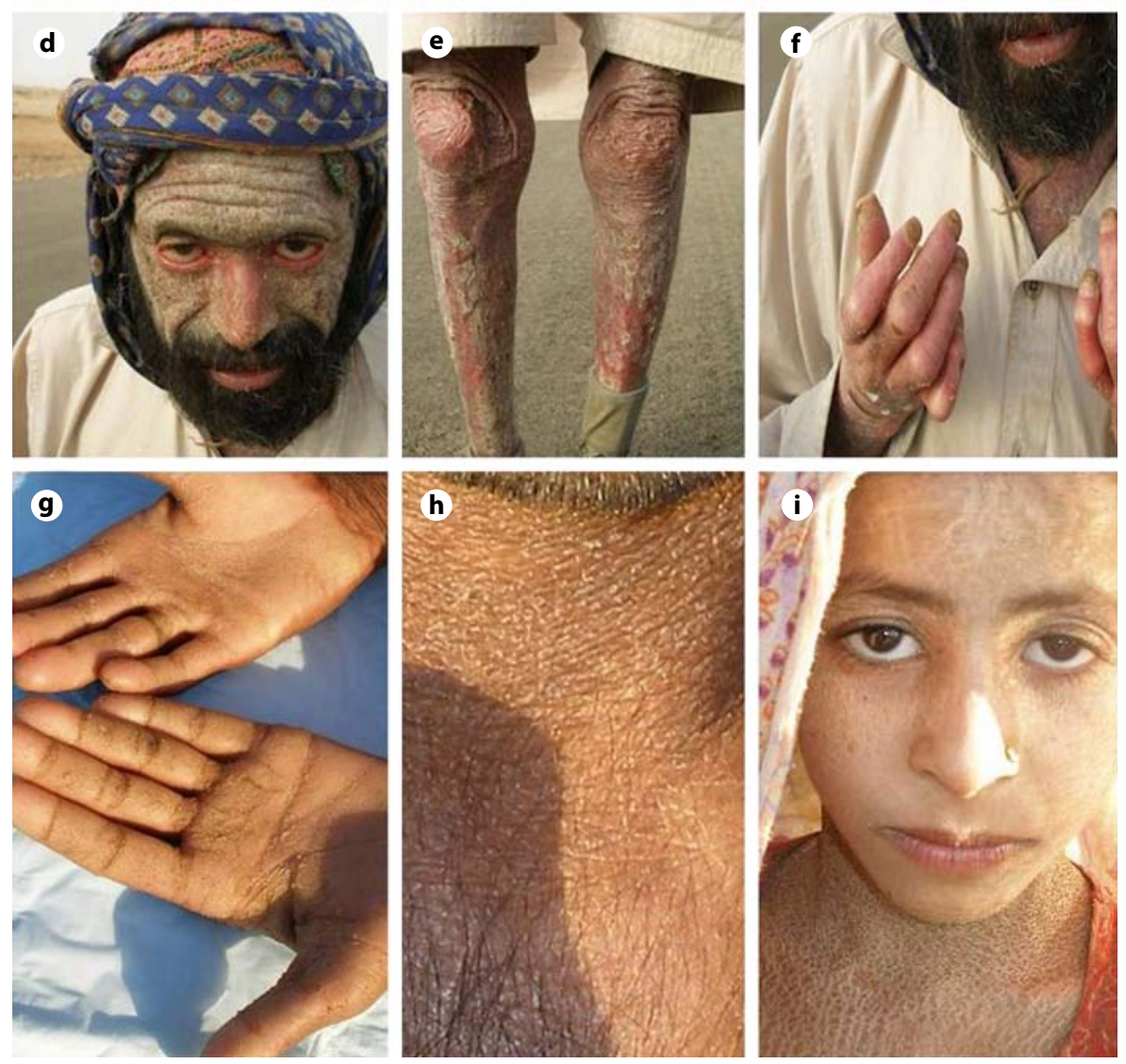

fine whitish scales involving the body and the face (fig. $2 \mathrm{~h}$ ), while other members had generalized reticulated brownish ichthyosis (fig. 2i) with an absence of the fine whitish scales over the face.

\section{Haplotype Analysis}

In order to determine if the phenotype was linked to any of the genes already known to cause ARCI, we performed a haplotype analysis using microsatellite markers around each of the known genes. PCR amplification was performed for both affected and unaffected individuals in the 2 families. The amplification conditions for each PCR were $94^{\circ} \mathrm{C}$ for $2 \mathrm{~min}$, followed by 35 cycles of $94^{\circ} \mathrm{C}$ for $30 \mathrm{~s}, 55^{\circ} \mathrm{C}$ for $30 \mathrm{~s}$ and $72^{\circ} \mathrm{C}$ for $30 \mathrm{~s}$, with a final extension at $72^{\circ} \mathrm{C}$ for $7 \mathrm{~min}$. The PCR products were then run on $8 \%$ polyacrylamide gel. Genotypes were assigned by visual inspection.

\section{Mutation Analysis}

All exons of the NIPAL4 gene and the filaggrin gene with adjacent sequences of exon-intron borders were amplified by PCR with primers and under conditions previously described $[2,10]$. The amplified PCR products were directly sequenced in an ABI Prism 310 automated sequencer using the ABI Prism BigDye Terminator Cycle Sequencing Ready Reaction Kit (PE Applied Biosystems). 


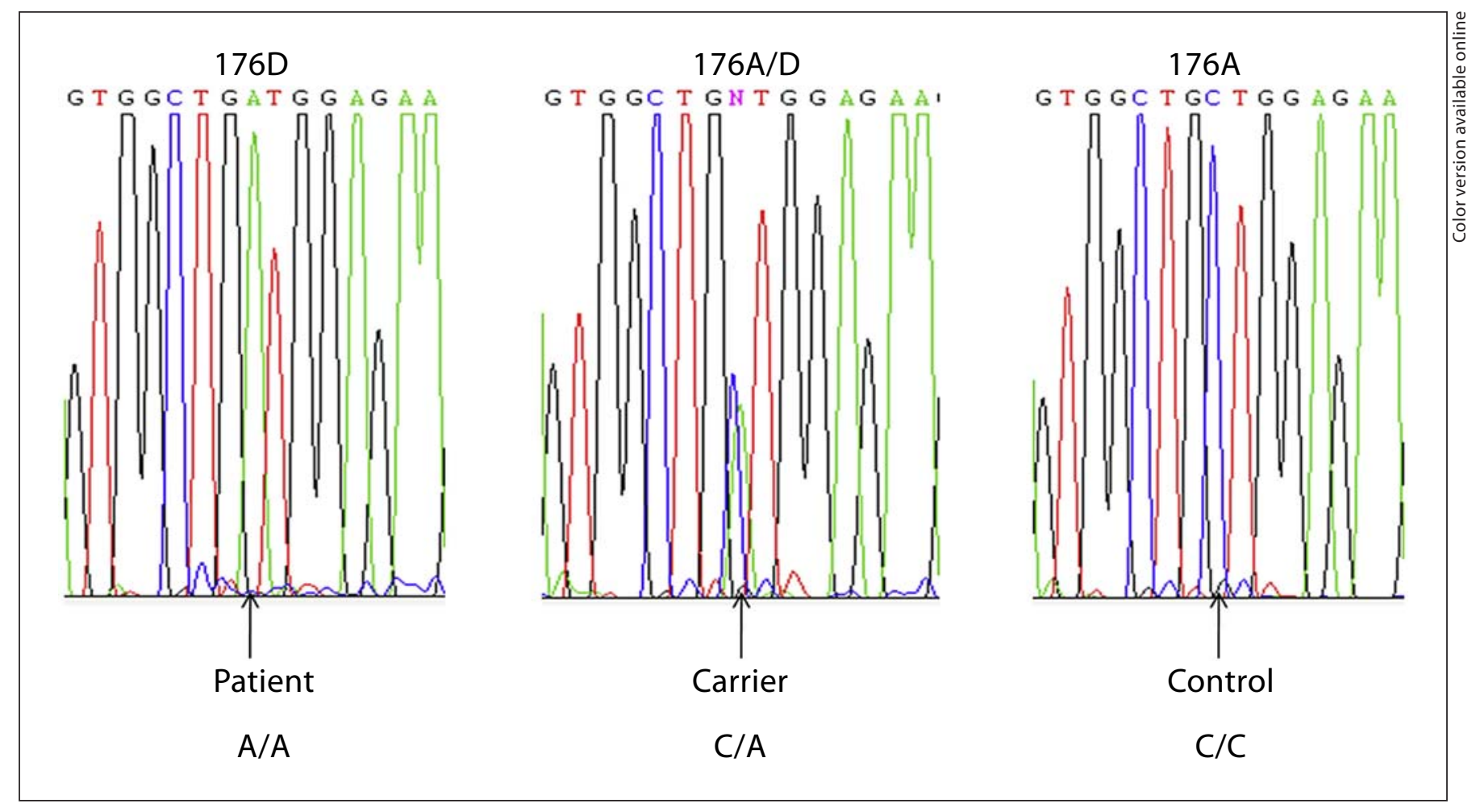

Fig. 3. Homozygous mutation characterized by $\mathrm{C} \rightarrow$ A transition leading to the substitution of aspartic acid (D) for alanine at codon $176(\mathrm{~A})$.
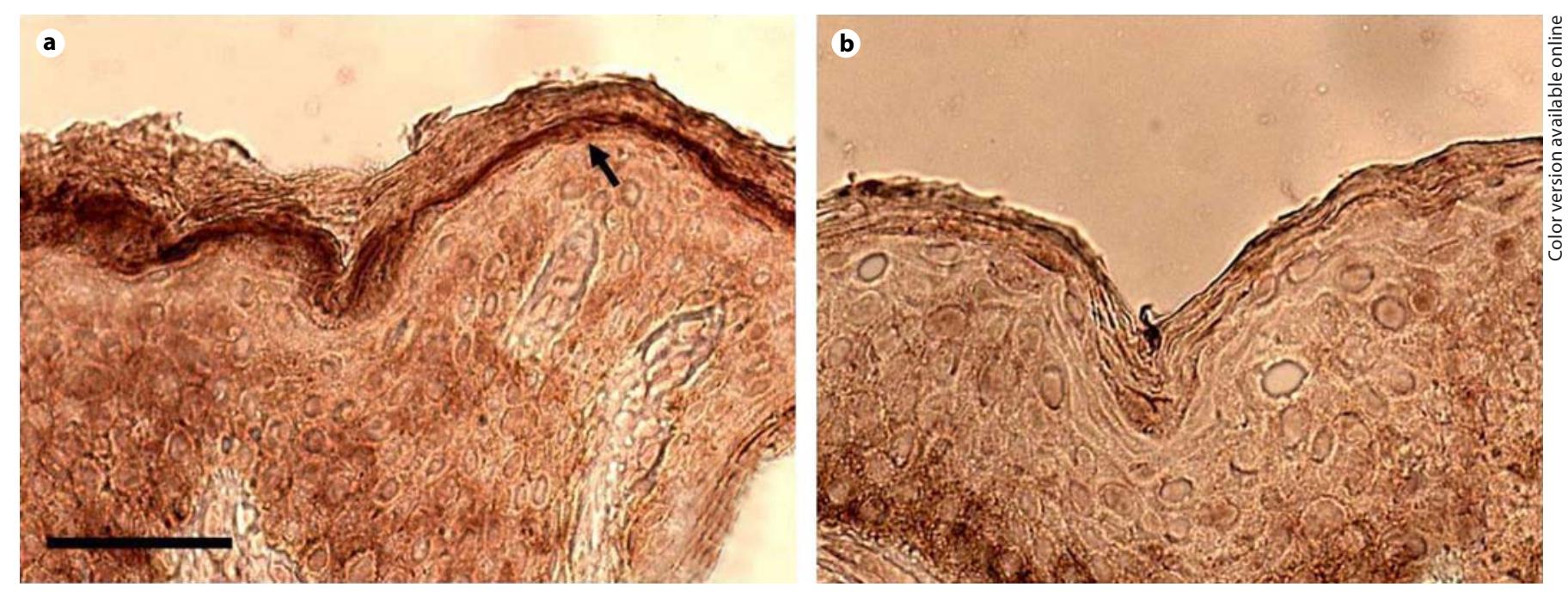

Fig. 4. a ISH reveals the expression of NIPAL 4 mRNA in the granular cell layer of the epidermis (arrow). b Negative control. 
In situ Hybridization

A fragment of the coding region of the human NIPAL4 cDNA (GenBank accession No. NM_001099287: 437-935 nt) was cloned into the $\mathrm{pCR}^{\circledR} \mathrm{II}-\mathrm{TOPO}$ vector (Invitrogen). The antisense and sense digoxigenin-labeled cRNA probes were synthesized from the linearized vectors with T7 and SP6 RNA polymerases (Roche Applied Science), respectively. Skin tissue of a healthy control individual was fixed with $4 \%$ paraformaldehyde-PBS at $4^{\circ} \mathrm{C}$ overnight. After a dehydration step with $30 \%$ sucrose-PBS, the tissue was frozen in optimal cutting temperature compound and sectioned on glass slides at a thickness of $7 \mu \mathrm{m}$. ISH was performed following the methods previously described [11].

\section{Results}

\section{Haplotype Analysis}

The haplotype analysis showed that both families were linked to the region encompassing the NIPAL4 gene on chromosome 5 (fig. 1), while neither family showed linkage to the other genes known to cause ARCI (data not shown).

Identification of Recurrent Mutation in NIPAL4 Gene

We performed a direct sequencing analysis for the NIPAL4 gene in both families. Affected individuals from both families were homozygous for the mutation c.527C $\rightarrow$ A in exon 4, which leads to a substitution of the amino acid alanine by aspartic acid, designated A176D (fig. 3). The uninvolved members were either heterozygous for the mutation or had the wild-type sequence consistent with autosomal recessive inheritance. No mutation was found in the filaggrin gene.

\section{NIPAL4 mRNA Expressed in Granular Cell Layer of Epidermis}

In order to localize the expression of NIPAL4 in human skin, we performed ISH studies in skin sections of a healthy control individual. The results demonstrate that the human NIPAL4 mRNA is predominantly expressed in the granular cell layer of the epidermis (fig. 4). The sense probe did not show positive signals (fig. 4).

\section{Discussion}

NIPAL4 is one of 6 genes that have been implicated in ARCI, and only a few families have been reported with mutations in this gene. Patients with NIPAL4 mutations resemble more the NBCIE subtype, despite the fact that some patients clinically look like LI patients. Clinically, they present with a collodion membrane, which is shed shortly after birth, although some may be born without this membrane [2]. They later develop generalized ichthyosis with erythema, fine whitish scales on the face and trunk, and larger brownish scales on the neck, buttocks and legs. In addition, patients develop palmoplantar keratoderma with fissures, sometimes associated with clubbing of the nails. Some reports describe ectropion, hypohidrosis, and patients with no or minimal erythema [12]. These features do not resemble the clinical picture of NBCIE, but are more similar to what is observed in LI. Therefore, the spectrum of clinical variability in patients with NIPAL4 mutation is very wide, with no consistent clinical features.

Patients with NIPAL4 mutations have been diagnosed from Mediterranean countries including Algeria, Turkey and Syria [2], and from Scandinavian countries including Sweden and Norway, in addition to a patient from the Faroe Islands and another from South America [12]. This report presents the first cases of NIPAL4 mutations from Pakistan.

Consistent with what has already been reported, we detected clinical variability even among members of the same family. These clinical features included severe skin dryness, which was common among all patients. In one family, the younger patients had clinical symptoms consistent with atopic dermatitis, which has not been reported to be a major manifestation of NIPAL4 mutations. This was associated with fine whitish scales over the face. The older affected member within the same family had ectropion, severe hyperkeratosis over the face and generalized ichthyosis with semiadherent whitish-to-yellowish scales on an erythematous base, associated with finger contractures and nail clubbing. These clinical manifestations represent a mixture of both LI and NBCIE phenotypes. In the second family, some members had a generalized reticulated brownish hyperkeratosis accentuated over the skin folds, associated with facial dyschromia, dryness and absence of the fine whitish scaling in addition to palmoplantar hyperkeratosis. On the other hand, some members of the same family did not have the reticulated brownish hyperkeratosis, but only had the fine whitish scales overlying an erythematous base over the body and face in addition to the palmoplantar hyperkeratosis. Despite having a common mutation, p.A176D, substantial clinical variation was evident. We therefore conclude that there is no genotype-phenotype correlation, and that environmental factors might play a major role in the different phenotypes.

Mutations in the NIPAL4 gene should be differentiated from other genes causing ARCI, such as the LOX 
and CYP4F22 genes. In general, patients with $L O X$ gene mutations and mainly those with $A L O X 12 B$ gene mutations are born with a collodion membrane, but later on they improve markedly, most of them achieving complete healing of their skin and others having a mild NBCIE phenotype [13]. On the other hand, patients with $C Y$ P4F22 are not born with collodion membranes, and this is very helpful to differentiate it from other genes causing ARCI, such as NIPAL4 and TGM1, but later on the patients start developing the LI phenotype [14], and thus, at that stage, it would be hard to differentiate it from the other genes causing LI.

The recurrent mutation, A176D located in exon 4, is the most common mutation reported in patients with NIPAL4 gene mutations. The fact that this mutation occurred in distinct populations suggests that it may be a hot spot for mutations at this site. In our case, both families showed the same disease-related haplotype, suggesting a common founder mutation in Pakistani families.

It has previously been found that levels of NIPAL4 cDNA are similar between affected patients and healthy controls [12], indicating that these mutations do not affect the stability of the transcript; therefore, the mechanism by which this mutation leads to the disease needs to be determined at the molecular level. It has been shown by electron microscopy that patients with the mutation A176D have abnormal lamellar bodies in the stratum granulosum and elongated perinuclear membranes [12]. Here, we showed by ISH that NIPAL4 is expressed in the granular layer of the epidermis, which is the site where the lamellar bodies are present. Our data provide evidence that NIPAL4 may be important for the formation of normal lamellar bodies, which are essential for the development of a normal skin barrier.

Although there were several individuals that had the clinical symptoms and features of atopic dermatitis, which were predominant over the ichthyosis, none of them had mutations in the FLG gene (data not shown), which is known to be involved in patients with atopic dermatitis [10]. Filaggrin is initially synthesized as profilaggrin, which is inactive. The subsequent processing of profilaggrin occurs in order to generate the active form, i.e. filaggrin monomers, which contribute to the skin barrier stability [15]. The disruption of profilaggrin processing by proteins from the lamellar bodies has previously been reported [16]. It has been shown in mice that matriptase, an enzyme found in lamellar bodies, is essential for the processing of profilaggrin, and that mutations in matriptase prevent the degradation of profilaggrin into its monomer form [16]. In humans, matriptase deficiency leads to ichthyosis, follicular atrophoderma and hypotrichosis (OMIM 602400). A second example is the Netherton syndrome (OMIM 256500), which clinically includes a combination of ichthyosis and atopic dermatitis in addition to other anomalies. This syndrome has a mutation in SPINK5, which codes for a protease inhibitor called LEKTI, located in the lamellar bodies [17]. Mutations in LEKTI have been shown to alter the processing of filaggrin [18]. The above evidence as well as the fact that syndromes that have alterations in filaggrin processing and filaggrin mutations, such as the Netherton syndrome and ichthyosis vulgaris (OMIM 146700), clinically develop ichthyosis and atopic dermatitis raise the possibility that the mechanism of action of NIPAL4 might be directly linked to filaggrin activity.

In conclusion, we detected a recurrent mutation in the NIPAL4 gene in 2 Pakistani families with ARCI and extended the spectrum of clinical features. Additionally, we demonstrated the expression of NIPAL4 in the granular cell layer, emphasizing its importance for establishing a normal epidermal barrier formation and furthering our understanding of the molecular function of the NIPAL4 gene.

\section{Acknowledgments}

We gratefully thank the families for having participated in this study. This study was supported by a USPHS (United States Public Health Service) NIH grant from the NIH/NIAMS (National Institute of Arthritis and Musculoskeletal and Skin Diseases; R01 AR44924) to A.M.C. and by an award of the Dermatology Foundation to Y.S.

References

Dermatology 2010;220:8-14
Farasat S, Wei MH, Herman M, Liewehr DJ, Steinberg SM, Bale SJ, et al: Novel transglutaminase-1 mutations and genotype-phenotype investigations of 104 patients with autosomal recessive congenital ichthyosis in the USA. J Med Genet 2009;46:103-111.

- 2 Lefèvre C, Bouadjar B, Karaduman A, Jobard F, Saker S, Özguc M, et al: Mutations in ichthyin a new gene on chromosome $5 \mathrm{q} 33$ in a new form of autosomal recessive congenital ichthyosis. Hum Mol Genet 2004;13:24732482 .

3 Griffiths WAD, Judge MR, Leigh IM: Disorders of keratinization; in Champion $\mathrm{RH}$, Burton JL, Burns DA, Breathnach SM (eds): Textbook of Dematology, ed 6. Oxford, Blackwell Science, 1998, pp 1483-1530. 
4 Eckl KM, de Juanes S, Kurtenbach J, Nätebus M, Lugassy J, Oji V, et al: Molecular analysis of 250 patients with autosomal recessive congenital ichthyosis: evidence for mutation hotspots in ALOXE3 and allelic heterogeneity in $A L O X 12 B$. J Invest Dermatol 2009;129: 1421-1428.

5 Lugassy J, Hennies HC, Indelman M, Khamaysi Z, Bergman R, Sprecher E: Rapid detection of homozygous mutations in congenital recessive ichthyosis. Arch Dermatol Res 2008;300:81-85.

6 Akiyama M: Harlequin ichthyosis and other autosomal recessive congenital ichthyoses: the underlying genetic defects and pathomechanisms. J Dermatol Sci 2006;42:83-89.

7 Hovnanian A: Harlequin ichthyosis unmasked: a defect of lipid transport. J Clin Invest 2005;115:1708-1710.

8 Madison KC: Barrier function of the skin: 'la raison d'être' of the epidermis. J Invest Dermatol 2003;121:231-241.

9 Elias PM: The epidermal permeability barrier: from the early days at Harvard to emerging concepts. J Invest Dermatol 2004;122: xxxvi-xxxix.
10 Palmer CN, Irvine AD, Terron-Kwiatkowski A, Zhao Y, Liao H, Lee SP, et al: Common loss-of-function variants of the epidermal barrier protein filaggrin are a major predisposing factor for atopic dermatitis. Nat Genet 2006;38:441-446.

-11 Shimomura Y, Wajid M, Petukhova L, Shapiro L, Christiano AM: Mutations in the lipase $\mathrm{H}$ gene underlie autosomal recessive woolly hair/hypotrichosis. J Invest Dermatol 2009;129:622-628.

12 Dahlqvist J, Klar J, Hausser I, Anton-Lamprecht I, Pigg MH, Gedde-Dahl T Jr, et al: Congenital ichthyosis: mutations in ichthyin are associated with specific structural abnormalities in the granular layer of epidermis. J Med Genet 2007;44:615-620.

13 Kurban M, Shimomura Y, Bahhady R, Ghosn S, Kibbi AG, Christiano AM: Nonsense mutation in the ALOX $12 B$ gene leads to autosomal recessive congenital ichthyosis in a Lebanese family. J Eur Acad Dermatol Venereol 2009, E-pub ahead of print.

14 Lefèvre C, Bouadjar B, Ferrand V, Tadini G, Megarbane A, Lathrop M, et al: Mutations in a new cytochrome $P 450$ gene in lamellar ichthyosis type 3. Hum Mol Genet 2006;15:767776.
15 Alef T, Torres S, Hausser I, Metze D, Türsen U, Lestringant GG, et al: Ichthyosis, follicular atrophoderma, and hypotrichosis caused by mutations in ST14 is associated with impaired profilaggrin processing. J Invest Dermatol 2009; 129:862-869.

16 Leyvraz C, Charles RP, Rubera I, Guitard M, Rotman S, Breiden B, et al: The epidermal barrier function is dependent on the serine protease CAP1/Prss8. J Cell Biol 2005;170:

487-496.
-17 Deraison C, Bonnart C, Lopez F, Besson C, Robinson R, Jayakumar A, et al: LEKTI fragments specifically inhibit KLK5, KLK7, and KLK14 and control desquamation through a $\mathrm{pH}$-dependent interaction. Mol Biol Cell 2007;18:3607-3619.

18 Hewett DR, Simons AL, Mangan NE, Jolin HE, Green SM, Fallon PG, et al: Lethal, neonatal ichthyosis with increased proteolytic processing of filaggrin in a mouse model of Netherton syndrome. Hum Mol Genet 2005; $14: 335-346$. 